\title{
ITERATES OF AN ABSOLUTELY CONTINUOUS OPERATOR
}

\author{
P. GHATAGE ${ }^{1}$
}

ABSTRACT. It is proved that the powers of an absolutely continuous polynomially bounded operator, the intersection of whose spectrum with the circle has zero measure, go to zero strongly. From this we deduce a simple treatment of the Nagy-Foias, result on completely nonunitary contractions.

In this note we generalize a theorem of Nagy and Foiaș [2, Chapter II, Proposition 6.7] to polynomially bounded operators. Our method also gives an elementary proof of their theorem by avoiding the use of dilation theory completely.

Recall that a polynomially bounded operator $T$ is said to be absolutely continuous if for every $x, y$ in $H$ there exists a representing measure $\mu$ for the functional $p \rightarrow(p(T) x, y)$ such that $\mu \ll m$ (= the Lebesque measure on the unit circle $\Gamma$ ).

Theorem. If $T$ is an absolutely continuous polynomially bounded operator for which $m(\sigma(T) \cap \Gamma)=0$, then $T^{n} \rightarrow{ }^{\text {st }} 0$ and $T^{*}{ }_{n} \rightarrow{ }^{\text {st }} 0$.

Proof. Let $M=\left\{h \in H:\left\|T^{n} h\right\| \rightarrow 0\right\}$, and let $P$ denote the projection on $M^{\perp}$. If $S=P T \mid M^{\perp}$, then $S^{n}=P T^{n} \mid M^{\perp}$ for $n \geq 0$ and, hence, $S$ is an absolutely continuous polynomially bounded operator. Moreover $\sigma(S) \cap \Gamma$ $C \partial \sigma(S) \cap \Gamma \subset \sigma(T) \cap \Gamma$ and $m(\sigma(S) \cap \Gamma)=0$. It is easy to see that for $h$ in $M^{\perp},\left\|S^{n} h\right\| \rightarrow 0$ only if $h=0$, and since $S$ is powerbounded, this implies that inf ${ }_{n \geq 0}\left\|S^{n} h\right\|=\mu(h)$ is strictly positive unless $h=0$. Thus there exists a one-to-one bounded positive operator $A$ on $M^{\perp}$ such that $(A h, k)=L\left\{\left(S^{n} h, S^{n} k\right)\right\}$, where $L$ denotes a generalized Banach limit on the set of bounded sequences. If $X$ denotes $A^{1 / 2}$, then $X S X^{-1}$ is an

Presented to the Society, November 6, 1973 under the title An extension of Nagy's theorem; received by the editors November 27, 1973 and, in revised form, May 20, 1974.

AMS (MOS) subject classifications (1970). Primary 47A99; Secondary 47A20.

Key words and phrases. Polynomially bounded, absolutely continuous, strong convergence. A8745.

1 Work supported by the National Research Council of Canada under Grant 
isometry on $R(X)$ and can be extended to an isometry $V$ on $M^{\perp}$. We claim that $\sigma(V) \cap \Gamma \subset \sigma(S) \cap \Gamma$. In fact, if $\|(S-\lambda) g\| \geq C\|g\|$ for all $g$ in $M^{\perp}$, then

$$
\begin{aligned}
\|(V-\lambda) X b\|^{2} & =\left\|\left(X S X^{-1}-\lambda\right) X b\right\|^{2}=\|X(S-\lambda) b\|^{2} \\
& =L\left\{\left\|S^{n}(S-\lambda) b\right\|^{2}\right\}=L\left\{\left\|(S-\lambda) S^{n} b\right\|^{2}\right\} \\
& \geq L\left\{C^{2}\left\|S^{n} b\right\|^{2}\right\}=C^{2}\|X b\|^{2} .
\end{aligned}
$$

Hence, by continuity for all $g$ in $M^{\perp}$, we have $\|(V-\lambda) g\| \geq C\|g\|$. Thus $m(\sigma(V) \cap \Gamma)=0$ and it follows that $V$ is a unitary for which $m(\sigma(V))=0$. Since $X S^{n}=V^{n} X$ for $n \geq 0$, it follows from the F. and M. Riesz theorem $[1$, p.47] that the spectral measure of $V$ is absolutely continuous. Thus we have a contradiction unless $M^{\perp}=(0)$. Since $T^{*}$ satisfies the same hypothesis as $T$, we have $T^{*} n \rightarrow{ }^{s t} 0$.

Corollary (Nagy-Foiaş). If $T$ is a c. n. u. contraction satisfying $m(\sigma(T) \cap \Gamma)=0$, then $T$ is in $C_{00}$.

Proof. We need only note that the singular part $T_{0}$ of $T$ vanishes. But the uniqueness of the representing measures $\sigma_{x, y}$ of $P \rightarrow\left(P\left(T_{0}\right)_{x}, y\right)$ allows us to extend $P \rightarrow P\left(T_{0}\right)$ to a representation $\Phi$ of $C(\Gamma)$, and this representation is still of norm one. Hence, $\left\|T_{0}^{-1}\right\|=\left\|\Phi\left(e^{-i \theta}\right)\right\| \leq 1$, so that $T_{0}$ is unitary.

We wish to thank the referee for suggesting this simple version of our proof for the Corollary.

\section{BIBLIOGR APHY}

1. K. Hoffman, Banach spaces of analytic functions, Prentice-Hall Series in Modern Analysis, Prentice-Hall, Englewood Cliffs, N. J., 1962. MR 24 \#A2844.

2. B. Sz.-Nagy and C. Foiaş, Analyse harmonique des opérateurs de l'espace de Hilbert, Akad. Kiadó, Budapest, 1967; English transl., North-Holland, Amsterdam; American Elsevier, New York, 1970. MR 43 \#947.

DEPARTMENT OF MATHEMATICS, UNIVERSITY OF GUELPH, GUELPH, ONTARIO, CANADA

Current address: Department of Mathematics, University of Toronto, Toronto, Ontario, Canada 